\title{
Urban-adapted mammal species have more known pathogens
}

\author{
Gregory F Albery* ${ }^{* 1}$; Colin J. Carlson ${ }^{2,3}$; Lily E Cohen ${ }^{4}$, Evan A. Eskew ${ }^{5}$; Rory \\ Gibb $^{6,7}$; Sadie J. Ryan ${ }^{8,9,10}$; Amy R Sweeny ${ }^{11}$; Daniel J Becker ${ }^{12}$
}

1. Department of Biology, Georgetown University, Washington D.C., 20007

2. Center for Global Health Science and Security, Georgetown University Medical Center, Washington, D.C., 20007

3. Department of Microbiology and Immunology, Georgetown University Medical Center, Washington, D.C., 20007

4. Icahn School of Medicine at Mount Sinai, New York, NY, 10029

5. Department of Biology, Pacific Lutheran University, Tacoma, WA, 98447

6. Centre for Mathematical Modelling of Infectious Diseases, London School of Hygiene and Tropical Medicine, London, UK.

7. Centre on Climate Change and Planetary Health, London School of Hygiene and Tropical Medicine, London, UK.

8. Quantitative Disease Ecology and Conservation (QDEC) Lab Group, Department of Geography, University of Florida, Gainesville, FL, 32610 USA

9. Emerging Pathogens Institute, University of Florida, Gainesville, FL, 32610 USA

10. School of Life Sciences, University of KwaZulu-Natal, Durban, 4041, South Africa

11. University of Edinburgh, Ashworth Laboratories, Edinburgh EH9

12. Department of Biology, University of Oklahoma, Norman, OK, USA

*gfalbery@gmail.com

\section{Abstract}

Mammals that regularly inhabit urban environments may have more frequent contact with humans and therefore host more known zoonotic pathogens. Here, we test this prediction using a consolidated dataset of phenotypic traits, urban affiliate status, and pathogen diversity, across 3004 mammal species. We show that urban-adapted mammals have more documented pathogens - and more zoonoses - even when considering a correlated suite of phenotypic, taxonomic, and geographic predictors. However, contrary to predictions, path analysis revealed that urban-adapted species do not host more zoonoses than expected given their total observed pathogen richness. We conclude that extended historical contact with humans has had a limited impact on the number of observed zoonoses in urbanadapted mammals. Instead, their greater observed zoonotic richness likely reflects either sampling bias due to greater cultural awareness and physical proximity to humans, or increased baseline pathogen diversity arising from the physiological and ecological consequences of urban living. 
Word Count: Abstract: 146 words; Main text: 4410 words

Number of figures: 3; Number of tables: 1; Number of references: 56

\section{Authorship Statement}

GFA and DB conceived the study, and GFA analysed the data and wrote the manuscript. All other authors offered thoughts on the analysis and commented on the manuscript.

\section{Data Accessibility Statement}

The code used here is available at https://github.com/gfalbery/UrbanOutputters. On acceptance, the data will be uploaded to the same repo, which will be archived on Zenodo.

\section{Introduction}

As the tide of emerging infectious diseases rises, it is becoming ever more important to identify and understand the drivers of zoonotic risk in wild animals (Jones et al. 2008; Keesing et al. 2010; Morse et al. 2012). Humans are rapidly altering patterns of wildlife disease through a combination of climate change and land conversion, both of which are expected to drive increased spillover of mammal pathogens into human populations (Jones et al. 2008; Keesing et al. 2010; Loh et al. 2015; Hassell et al. 2017; Carlson et al. 2020a; Cohen et al. 2020; Gibb et al. 2020). Urban environments in particular are expected to facilitate zoonotic emergence in wildlife (Keesing et al. 2010; Hassell et al. 2017; Becker et al. 2018; Murray et al. 2019; Werner \& Nunn 2020), through a combination of impaired immune systems fed by anthropogenic resources (Becker et al. 2015, 2018) and greater pollution (Becker et al. 2019), and increased proximity to humans (Hassell et al. 2017; Albery $\&$ Becker 2021). This combination of factors is likely to become even more problematic in the future as the world's population continues to grow and urbanize (Seto et al. 2012; Chen et al. 2020; Gao \& O'Neill 2020). Previous meta-analyses have uncovered associations between elevated stressors and greater pathogen burdens or diversities in animals inhabiting urban settings (Murray et al. 2019; Gibb et al. 2020; Werner \& Nunn 2020), with a general expectation that the urban environment will weaken the immune response. However, these studies usually compare high-resolution datasets involving urban and non-urban populations of the same species, and are therefore based on relatively few examples spread across a range of vertebrates and non-vertebrates. Verifying whether urban-adapted mammal species exhibit greater zoonotic risk in a broad-scale, pan-mammalian analysis could strengthen the support for this phenomenon, informing the design of pathogen sampling regimes, and supporting efforts to mitigate zoonotic disease risk in human populations.

If urban-adapted mammals present a greater zoonotic risk, they may be key to understanding a long-held observation: that fast-lived host species (i.e., those that favour reproduction over survival) tend to disproportionately source zoonotic pathogens (Keesing et 
al. 2010; Ostfeld et al. 2014; Albery \& Becker 2021). Associations between fast life history and zoonotic risk are commonly proposed to be mediated by inherent immune differences (e.g. (Ostfeld et al. 2014; Han et al. 2020)); however, immunity is only one factor contributing to a species' zoonotic potential, and between-host, exposure-related processes may also play a role (Albery \& Becker 2021). A recent study revealed that fast-lived species are also more likely to be urban-adapted (Santini et al. 2019), which could disproportionately expose them to the immunity-inhibiting effects of urban environments, while also placing them in closer proximity to high-density human populations (Albery \& Becker 2021). Even within urban environments, small, fast-lived species are more likely to be pests and commensals with access to the interior of human dwellings (Masi et al. 2010; Hulme-Beaman et al. 2016). In sum, these trends could lead fast-lived species to more often transmit pathogens to humans. As such, characterizing (zoonotic) pathogen diversity in synanthropic animals is an important step towards unravelling the mechanisms driving observed patterns of zoonotic risk.

Here, we use several large-scale, publicly available databases to investigate whether urbanaffiliated mammal species have more zoonotic pathogens, and whether they source more zoonoses than expected, given their overall pathogen diversity. We anticipated that species capable of adapting to urban settings would host a higher diversity of pathogens owing to greater susceptibility and more intense sampling, and that a disproportionately high number of these pathogens would be known to be zoonotic as a result of their greater historical contact with humans. We further expected that urban affiliation status would account for some variation in life history effects, implying that fast-lived species more often source zoonoses as they more likely inhabit urban environments in close proximity to humans (summarized in Table 1).

\section{Proximate mechanism}

Urban $\rightarrow$ more zoonoses specifically

Urban $\rightarrow$ more pathogens $\rightarrow$ more zoonoses

\section{Ultimate mechanism}

More historical contact with human populations have allowed greater spillover rates

Urban environments alter host ecology or physiology, increasing pathogen diversity
Urban $\rightarrow$ more known pathogens $\rightarrow$ more zoonoses
Urban-adapted mammals have been more heavily sampled

Table 1: Potential mechanisms driving greater zoonotic diversity in urban mammal species. 


\section{Methods}

\section{Data sources}

Phylogeographic data. We used the PanTHERIA dataset (Jones et al. 2009) as a

backbone for mammal taxonomy and phenotypic traits such as body mass. Phylogenetic data were derived from a mammalian supertree (Fritz et al. 2009), as used for several hostvirus ecology studies (e.g. Olival et al. 2017; Albery et al. 2020; Becker et al. 2020). The tree's phylogenetic distances between species were scaled between 0 and 1. Geographic data were taken from the IUCN species ranges (IUCN 2019). For each species, we calculated total range area by adding together the areas for the $25 \mathrm{~km}$ raster cells in which they were present.

To derive a measure of study effort, which often explains substantial variation in pathogen diversity (Olival et al. 2017; Mollentze \& Streicker 2020), we conducted systematic PubMed searches to identify how many publications mentioned a given mammal species, following previous methodology (Becker et al. 2020a). Domestication status used a sensu lato definition based on whether a species has ever been partially domesticated, coded as a binary variable. For example, despite being widespread in the wild, the European red deer (Cervus elaphus) is coded as "Domestic" because it is often farmed, notably in New Zealand (Mason 1994). Because we were investigating spatial distributions of species (see above), fully domesticated species that do not exist in the wild (e.g. cattle, Bos taurus) were generally excluded due to their absence from the IUCN species ranges. To investigate whether dietary flexibility could affect pathogen diversity, following previous methodology (Santini et al. 2019), we derived a measure of diet diversity by calculating a Shannon index from the EltonTraits database proportional diet contents (Wilman et al. 2014).

Life history data. To investigate how host life history variation affects pathogen richness, we used a previously-published, mass-corrected principal components analysis (PCA) of life history variation across mammal species (Plourde et al. 2017). The first two principal components (PCs) from this analysis, which explained $86 \%$ of variation in six life history traits (Plourde et al. 2017), were used as explanatory variables in our models. The six life history traits were gestation length, litter size, neonate body mass, interbirth interval, weaning age, and sexual maturity age. PC1 explains $63 \%$ of the variance in the six traits, representing a generalisable slow-fast axis. PC2 explains $23 \%$ of variance in these traits and represents investment in gestation time and larger offspring. Both PCs were available for all mammals in our dataset. We coded the PCs such that increasing values corresponded to "faster" life history (i.e., favouring greater reproduction over survival). 
Urban adaptation data. We identified each species' habitat preferences using a published database of long-term urban affiliation status in mammals that have been observed affiliated with urban environments in long-term contexts (Santini et al. 2019). This dataset was compiled using literature searches to identify species that were observed inhabiting urban environments; species are either coded as a "visitor" or a "dweller", based on whether they rely fully on urban environments to survive and reproduce (dweller) or whether they continue to rely on non-urban resources (visitor). This approach distinguishes our analysis from previous studies (e.g. Gibb et al., 2020): we use a strict definition of "urban-adapted" species, defining them as "mammals that survive, reproduce, and thrive in urban environments," rather than basing urban status purely on survey records collected in urban settings. All species that were in PanTHERIA but were not in the urban adaptation dataset were coded as "non-urban". We used urban affiliation as a binary variable, coding species as 0 or 1 depending on whether it was in the urban affiliate dataset. Overall, 180 species in our dataset were coded as a 1, denoting that they had been observed living off urban resources.

Host-pathogen association data. Pathogen diversities were derived by combining four large-scale datasets of host-pathogen associations, collected through a combination of web scrapes and systematic literature searches (Wardeh et al. 2015; Olival et al. 2017; Stephens et al. 2017; Shaw et al. 2020). These contained a range of pathogen groups, including viruses, bacteria, protozoa, fungi, helminths, and arthropods. In this conjoined dataset, hostpathogen associations were counted according to demonstrated compatibility: that is, if a host species had ever been discovered infected with a given pathogen, it was coded as a 1 , and all undemonstrated associations were assumed absent. We cleaned the pathogen names with the R package taxize (Chamberlain \& Szöcs 2013) to ensure that no hostpathogen associations were counted twice, leaving 17,098 unique host-pathogen associations.

From this conjoined dataset, we derived the following traits for each mammal host species in our dataset: 1) Total pathogen richness: the number of unique pathogen species known to infect a given host species; 2) Zoonotic pathogen richness: the number of these pathogens that has also been observed to infect humans in our dataset. All analyses were repeated for overall pathogen numbers (e.g., total number of zoonoses across all pathogen groups) and for specific pathogen subgroups (viruses, bacteria, protozoa, fungi, helminths, and arthropods).

For each analysis, to facilitate model fitting, we eliminated species for which there were missing data and then removed all host orders for which there were fewer than 20 species, or for which fewer than $1 \%$ of species had one or more known pathogens. Leaving these 
taxa in did not notably alter fixed effects estimates generally, but generated unlikely estimates for order-level effects. When combining the phenotypic, urbanisation status, and viral datasets, any species with no known pathogen associations was coded as a zero (i.e., a pseudoabsence), under the assumption that species with no known pathogens are still informative of variables associated with low pathogen richness (Albery \& Becker 2021).

\section{Models}

Base model. To analyse associations between urban affiliation status and pathogen richness, we used Generalised Linear Mixed Models (GLMMs) inferred using Integrated Nested Laplace Approximation (INLA) (Lindgren et al. 2011; Lindgren \& Rue 2015). We used two response variables with a negative binomial distribution: total pathogen richness and zoonotic pathogen richness, where the second value was a subset of the first. Explanatory variables included: Citation number $(\log (x+1)$-transformed); Host order (7 levels: Artiodactyla, Carnivora, Chiroptera, Lagomorpha, Primates, Rodentia, Soricomorpha); Urban affiliation status (binary; non-urban/urban); range area (continuous, log-transformed, defined above); Phylogenetic distance from humans (continuous, scaled 0-1); Body mass (continuous, log-transformed); Domestication status (binary); and two life history principal components (PC1 and PC2; continuous, taken from Plourde et al. 2017). We also applied these models to each pathogen subset to assess the generality of our parameter estimates.

Interaction model. We then compared the base model with one including an interaction between host order and urban adaptation status to investigate whether this urban effect varied taxonomically. We used the Deviance Information Criterion (DIC) to measure model fit, with a threshold change ( $\triangle \mathrm{DIC})$ under 5 denoting competitive models.

Multivariate models. To investigate whether urban affiliation status had different effects for the richness of different pathogen types, we fitted two multi-response models using the MCMCgImm package (Hadfield 2010): one for overall richness and one for zoonotic richness. These models used each of the six pathogen groups as response variables, and included the same fixed effects, with different (but correlated) slopes for each response. Comparing the model's estimates for the effect of urban affiliation for each pathogen allowed us to ask whether specific pathogen groups are significantly more likely to be associated with urbanisation status than others.

Zero-inflated models. To investigate whether pseudoabsences were disproportionately altering our results, we ran zero-inflated models of pathogen and zoonotic richness again using MCMCgImm to control for processes that specifically generate zero-counts. These models generate two estimates for each explanatory variable: 1) the effect on the probability that a species' pathogen count is greater than zero ("zero-inflation") and 2) the effect on 
pathogen count greater than zero when accounting for this effect ("Poisson"). Importantly, the Poisson component of this model generates some zeroes itself, which improves upon similar models (e.g. hurdle models) in which all zeroes must be produced by the inflation term. This model allows us to identify whether, for example, urban species are simply more likely to have one or more known pathogens, rather than having a greater overall known pathogen richness, and whether our choice to code mammals with no known pathogens as zero-counts would influence the results.

Path analysis. To investigate whether urban mammals had a disproportionately high zoonotic richness when accounting for overall pathogen richness, we fitted a path analysis (Shipley 2009) with zoonotic richness as the ultimate response variable, log(overall richness +1 ) as an explanatory variable, and every other explanatory variable described above. We took 1000 random draws from the posterior distributions of 1 ) the effect of urban affiliate status on overall pathogen diversity; 2) the effect of urban affiliate status on zoonotic richness; and 3) the effect of overall richness on zoonotic richness. This approach allowed us to identify whether urban affiliation had a significant positive effect on zoonotic richness when accounting for its effect on pathogen richness as a whole, informing us as to whether a disproportionate number of urban mammals' known pathogens are known zoonoses.

Spatial model. Observed pathogen diversity in mammals is highly spatially heterogeneous at a global level (Allen et al. 2017; Olival et al. 2017; Carlson et al. 2020b), while the diversity of known urban-affiliated species is heavily biased towards North America and Eurasia (Santini et al. 2019). Both are driven by a combination of geographic variation in sampling effort as well as biotic and abiotic factors. To control for these spatial heterogeneities, we fitted spatial explanatory variables using three approaches. First, we 1) used a stochastic partial differential equation (SPDE) effect in INLA (Lindgren et al. 2011; Lindgren \& Rue 2015). This effect used species' geographic centroids in their IUCN ranges to control for spatial autocorrelation in the response variable according to Matern correlation, where species that were closer in space would be predicted to have similar numbers of known pathogens as a result of sampling bias and biological factors. We first fitted one spatial field to the whole dataset to look for overall spatial structuring, and we then allowed this spatial effect to vary for urban and non-urban species to investigate whether the distribution of known richness varies between these hosts. We also 2) incorporated species' presence on each of five continents (Eurasia, Africa, North America, South America, and Oceania) as binary variables and 3) added absolute latitude (i.e. distance from the equator). For the latter two approaches, we also fitted an interaction with urban status to investigate whether the effect of urban affiliation status varied across space (which could be the case if, for example, temperate cities are more heavily sampled than tropical cities). 

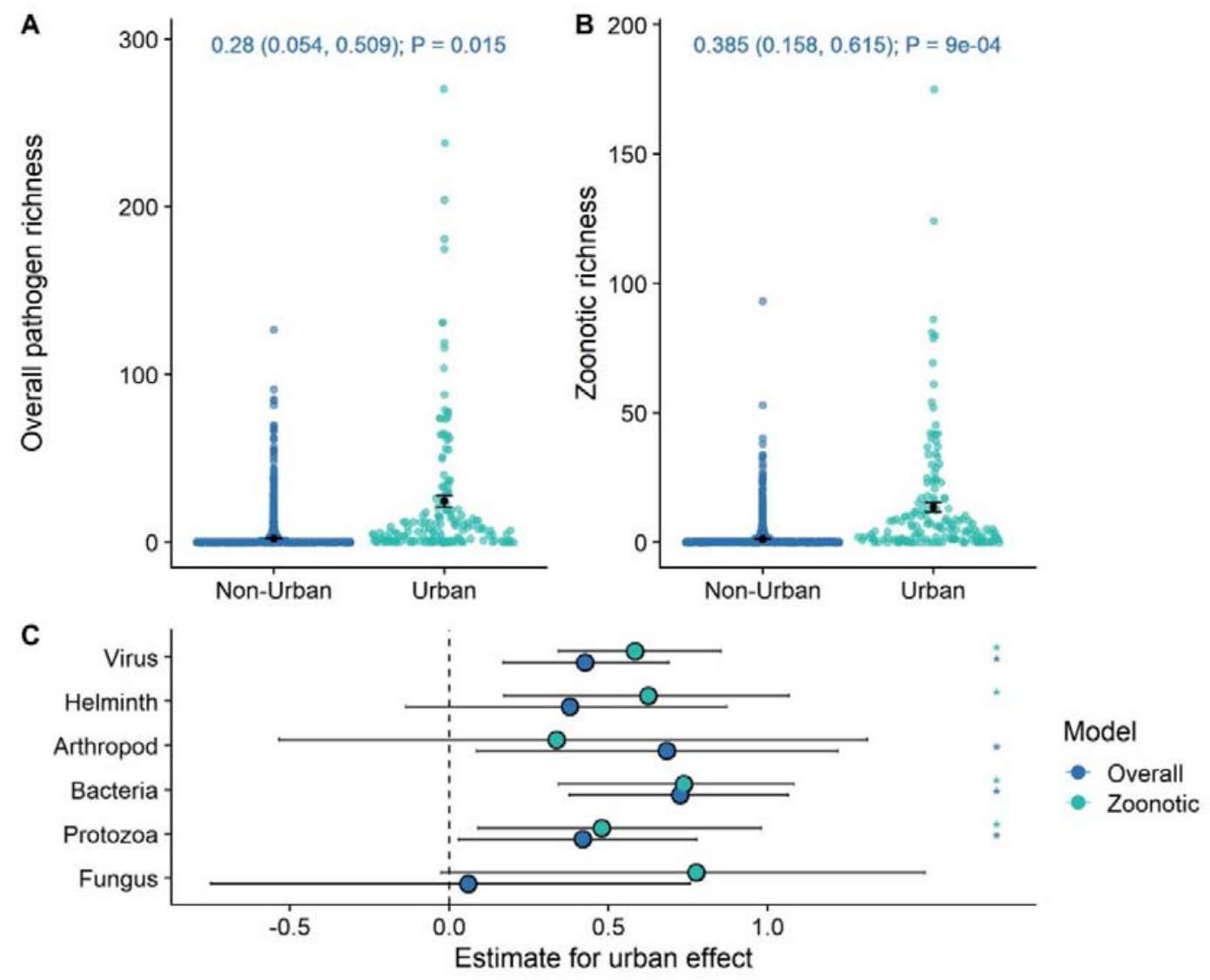

Figure 1: Urban-adapted mammals have more known pathogens $(A)$ and zoonoses specifically (B). Each point represents a mammal species, stratified by species that can capitalize on urban environments and those that do not. The $Y$ axis represents the species' known pathogen diversity. Black dots and error bars represent raw group means and standard errors, respectively. Displayed at the top of each panel are effect sizes for the between-group difference, 95\% credibility intervals (in brackets), and $P$ values (based on proportional overlap with 0 ), taken from our GLMMs including other explanatory variables. (C) Model effect sizes and 95\% credibility intervals for the 'MCMCglmm ` multivariate model, demonstrating that estimates for the effect of urban affiliation on pathogen richness did not differ notably across pathogen groups.

\section{Results}

Our models broadly supported our prediction that urban-adapted mammals would have greater pathogen richness. As expected, our first model set revealed that urban mammals have more known pathogens (Figure 1). This was the case for pathogens overall, and for arthropods, bacteria, protozoa, and viruses (Figure SI1). Furthermore, urban mammals had more zoonoses specifically, both overall (Figure 1) and for bacteria, helminths, protozoa, and viruses (Figure SI2). However, our path analyses revealed that, when accounting for a direct 
effect of pathogen richness, urban affiliation had no notable effect on zoonotic richness; in fact, the estimated effect was slightly negative (Figure 2). The indirect effect of urbanisation on zoonotic diversity acting through pathogen diversity was positive, substantial, and significant (effect $+0.401 ; 95 \%$ credibility interval 0.116-0.749; $P=0.004$; Figure 2 ). Taken together, these results demonstrate that positive effects of urban affiliation on zoonotic diversity act largely through greater known pathogen diversity overall, rather than by disproportionately elevating zoonotic pathogen richness specifically. Our secondary models revealed no improvement when urban status interacted with host order, demonstrating that the effect of urban affiliation on pathogen diversity and zoonotic risk did not vary between mammal taxa ( $\triangle \mathrm{DIC}<5$ relative to the base model). Similarly, the multivariate models revealed concordance between urban estimates across pathogen subtypes and implied that the urban effects were not being driven by specific groups of pathogens (Figure 1C). Finally, our zero-inflated models demonstrated strong urban biases for the count component rather than the inflation component, implying that these results are not being disproportionately driven by excess zeroes produced by the inclusion of pseudoabsences (Figure SI6).

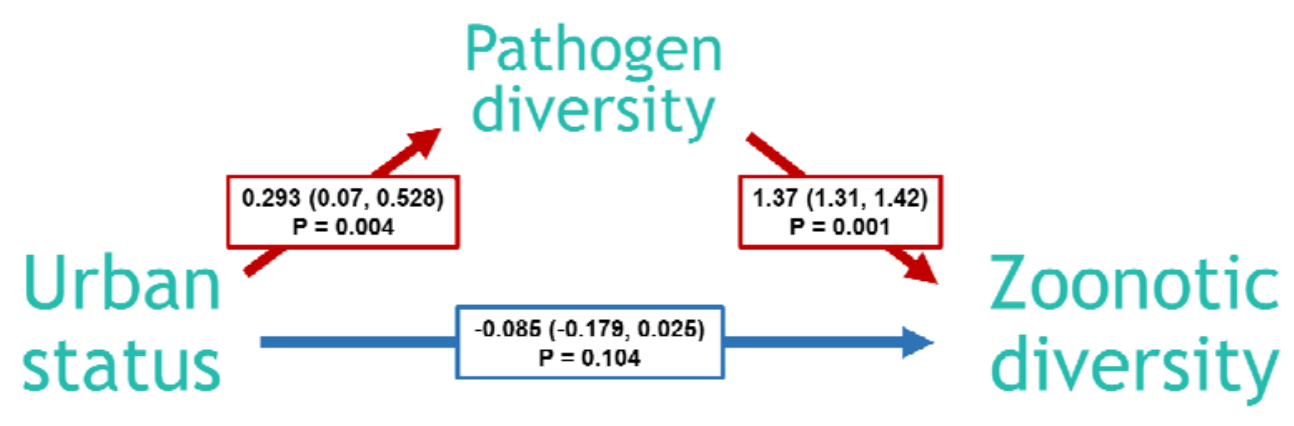

Figure 2: Path analysis revealed that urban-adapted mammals do not have more zoonoses than expected given their overall pathogen diversity. Arrows denote hypothesised causal relationships. Red lines represent positive effects and blue lines represent negative effects. Other variables were included in the component linear models, but are not displayed in this figure for clarity. Labels display the model effect estimates on the log link scale, with 95\% credibility intervals in brackets, and $\mathrm{P}$ values based on proportional overlap with 0 .

Adding the centroid-based SPDE effect improved model fit $(\triangle D I C<-150$ relative to the base model), but did not notably alter fixed effect estimates, including the effect of urban status (Figure 3A). Moreover, the model with different spatial fields for urban and non-urban species was not an improvement on the overall SPDE model $(\triangle \mathrm{DIC}=+14.35$ relative to the SPDE model). These findings imply that our results were robust to geographic variation in pathogen richness, and revealed strong spatial patterns (Figure $3 \mathrm{C}$ ). There was a substantial positive effect of absolute latitude, revealing greater known pathogen diversities 
in temperate regions (Figure 3B). There was also substantial between-continent variation (Figure 3B): North America was associated with greatest pathogen diversity, followed by Africa, then Eurasia, South America, and Oceania.

A
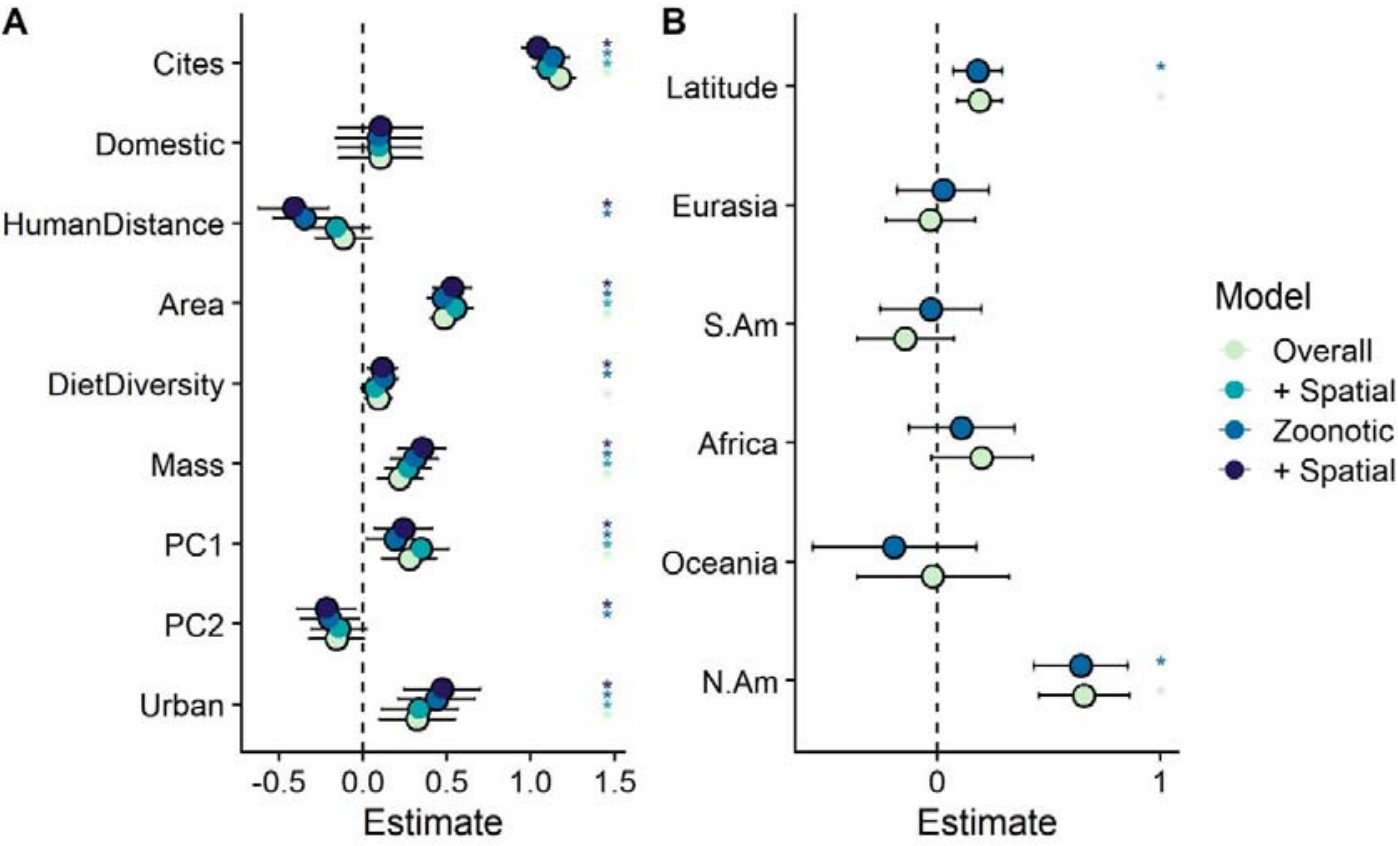

C

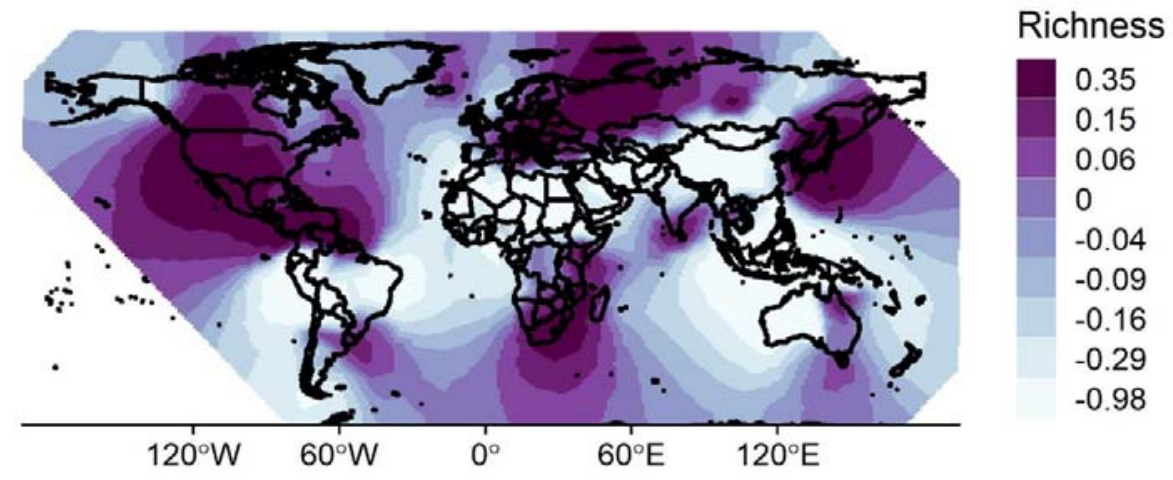

Figure 3: Model effect estimates and spatial effects. (A) Fixed effects from the models for overall pathogen richness and zoonotic richness, excluding order-level effects. (B) Fixed effect estimates from the non-spatial GLMMs for overall pathogen richness and zoonotic richness. Points represent the mean of the posterior effect estimate distribution from the GLMMs; error bars represent the $95 \%$ credibility intervals. Asterisks denote estimates that were significantly different from zero. Order-level effects have been left out for clarity; see the Figures SI4-5 for full model effect estimates. (C) Spatial distribution of the SPDE random effect, identifying hot- and coldspots of pathogen richness when non-spatial fixed effects (all effects except latitude and continent) are taken into account. Darker colours correspond to greater pathogen richness. 
We also uncovered effects of a range of other species traits (Figure 3A). Most notably, faster life history was associated with greater (zoonotic) pathogen diversity, according to PC1 (Figure 3A). However, in the path analysis model, the effect of life history on zoonotic richness was supplanted by the inclusion of overall pathogen richness (Figure SI3). This finding demonstrates that, as with urban affiliation status, life history is associated with greater overall pathogen richness rather than zoonotic richness specifically. Additionally, citation number was strongly positively associated with overall pathogen richness, overall zoonotic richness, and their equivalents for all pathogen subgroups (Figure 3A, Figure SI45). Diet diversity was positively associated with overall pathogen richness and with zoonotic richness (Figure $3 \mathrm{~A}$ ). Phylogenetic distance from humans was negatively associated with zoonotic richness overall (Figure $3 \mathrm{~A}$ ), with zoonotic richness of viruses, helminths, and bacteria, and with overall richness of viruses and protozoa; however, it was positively associated with overall richness of arthropods (Figure SI4-5). Greater range area was associated with increased (zoonotic) pathogen richness overall (Figure $3 \mathrm{~A}$ ) and for a range of models (Figure SI4-5). Finally, domesticated species had more zoonotic helminths and protozoa (Figure SI4-5), but had no difference in terms of overall richness (Figure 3A).

\section{Discussion}

Using an aggregated dataset of mammal species' traits and known pathogen associations, we demonstrate that urban-affiliated mammal species have more known pathogens, and more zoonotic pathogens as a result. This finding agrees with a growing body of evidence that human-dominated and urban landscapes encourage greater burdens of wildlife disease (Becker et al. 2015, 2019; Hassell et al. 2017; Murray et al. 2019; Carlson et al. 2020a; Gibb et al. 2020; Werner \& Nunn 2020). Surprisingly, we found that urban mammals' zoonotic richness was not greater than expected given their observed pathogen richness, confirming that a given species' propensity to host zoonoses is fundamentally a product of its overall pathogen richness (Mollentze \& Streicker 2020). This finding builds on recent work showing that wild animals inhabiting human-managed landscapes have more pathogens overall and more zoonoses (Gibb et al. 2020); however, we here used a much broader dataset of urbanaffiliated mammals and applied a strict definition of urban adaptation based on long-term resource use and fitness in urban landscapes (Santini et al. 2019). Given that overall pathogen richness is the primary driver of zoonotic richness, our findings do not support our main prediction that urban-adapted species host more known zoonoses because they have had more historical contact with humans, allowing more of their pathogens to spill over (Albery \& Becker 2021). The reason for urban mammals' greater pathogen richness remains uncertain, and many questions still linger about the drivers of zoonotic diversity in urban 
wildlife. Most pressingly, why is it so difficult to detect the signal of human-wildlife contact, when this should be a major component of a given species' zoonotic potential?

We offer two non-exclusive explanations for the greater pathogen diversity of urban species: sampling biases and urban effects on host biology. Fundamentally, urban mammals' tendency to live closer to humans may inevitably leave them more likely to be examined for pathogens, zoonotic or not. A similar bias may drive the high pathogen diversity of domestic animals (Wells et al. 2018, 2020). Notably, urban status was much more important than domestication status in our models, which implies that synanthropic animals are more likely to be examined for pathogens regardless of whether humans consider them to be useful. The question of how to account for research effort in host-pathogen ecology is an enduring one, and many studies use citation counts as we did here (Olival et al. 2017; Guy et al. 2019; Albery et al. 2020; Becker et al. 2020a; Gibb et al. 2020). The detection of this additional implied urban bias suggests that citations capture only one component of sampling bias, and researchers working on zoonoses should endeavour to identify similar measures of potential bias in the future. Furthermore, our findings held when we incorporated spatial effects, and we did not detect any spatial or taxonomic variation in the strength of the urban effect, implying that this bias is independent of geographic and taxonomic biases in sampling effort (Allen et al. 2017; Olival et al. 2017) and of known urbanisation status (Santini et al. 2019). To investigate whether urban species present fundamentally different opportunities for pathogen sampling, future studies could compare accumulation and rarefaction curves for pathogen diversities of urban and non-urban species to examine whether they saturate at different levels, potentially informing the value of focusing on urban species for future pathogen surveillance. Regardless, the evidence presented here suggests that urban species are well-sampled for pathogens, and this potential urban bias should be considered in future species-level analyses of zoonotic risk.

Urban environments could increase pathogen diversity through effects on host immunity, behaviour, and demography, such that urban species' greater pathogen count reflects real biological variation rather than artefacts of sampling bias. Anthropogenic pollutants, altered nutrition, and greater host densities in urban environments may weaken host immune systems, leading to greater burdens and diversities of pathogens (Becker et al. 2018, 2019; Murray et al. 2019; Werner \& Nunn 2020). Similarly, greater host densities and resource concentrations could facilitate elevated rates of density-dependent pathogen transmission within and between species, rendering urban-affiliated species more likely to maintain pathogens and resulting in greater observed pathogen diversity (Lloyd-Smith et al. 2005). However, there is some evidence that urban wildlife might exhibit stronger immunological resistance (Hwang et al. 2018; Strandin et al. 2018; Cummings et al. 2020), which would be 
expected to have the opposite effect on pathogen diversity. Notably, the bias towards urban mammals did not vary notably across pathogen subgroups, implying that an immune bias would have to act similarly on a diverse set of pathogens, from viruses to arthropod ectoparasites. It seems more likely that this homogeneity would be achieved through sampling processes, rather than ubiquitously increasing their susceptibility and diversity across pathogen groups. Unfortunately, the field is generally lacking in large-scale crossspecies analyses of immune function that would be required to differentiate these possibilities (Albery \& Becker 2021; but see Downs et al. 2020a, b). Ideally, future analyses incorporating life history, habitat preference, immunity, and pathogen diversity may be better able to differentiate the mechanisms underlying these species' zoonotic risk (Albery \& Becker 2021).

Because our analysis examined between-species variation, the underlying phenotypes of mammal species that allow them to colonise urban environments (e.g., small size, fast life history, diet generalism; Santini et al. 2019) could produce our results by inherently encouraging a greater pathogen diversity, rather than urban affiliation driving greater pathogen diversity per se. This problem could be addressed through broad meta-analyses of within-species variation in immunity and disease across urbanisation gradients, which have thus far examined a relatively small number of species and focused primarily on pathogens rather than immunity (Murray et al. 2019; Werner \& Nunn 2020). However, our models included a number of fixed effects (body mass, taxonomy, life history, diet diversity) that were previously found to influence urban adaptation probability (Santini et al. 2019), and revealed an urban bias to pathogen diversity that was additional to these effects. As such, it appears more likely that urban affiliation itself is generating these patterns of pathogen diversity, rather than underlying species-level drivers of urban adaptation.

To achieve broad insights into the drivers of pathogen diversity, this study was conducted at the species level, and with a minimum compatibility filter: that is, a species was considered a host of a given pathogen if it was observed with said pathogen at any point in the known literature, and richness was calculated as the sum of these associations across pathogen subgroups. While studies of pathogen diversity are common in macroecology, this deliberately narrow scope limits inference about a range of relevant processes including host competence (i.e., species' ability to transmit pathogens; Becker et al. 2020b), prevalence of the pathogen in the host populations, host population density, and, therefore, the rate of spillover. These are all important components of a species' zoonotic risk, and some hosts undoubtedly present substantial zoonotic risk despite having relatively low known pathogen diversity. For example, prairie dogs (Cynomys ludovicianus) only have five known pathogens in our dataset, yet they are a widespread and abundant species, and may play an important 
role in epizootics outbreaks of plague (Yersinia pestis) in North America (Hanson et al. 2007). Given this disparity, although the two are doubtless correlated, it is unclear how closely a species' zoonotic diversity should correlate with their rate of spillover. For policy and One Health audiences, we caution that our analysis does not necessarily offer insights into the relative frequency or rate of spillover events, or the potential severity of zoonotic outbreaks, in urban environments. Although our analysis reveals that urban mammals do not source a disproportionate number of different zoonotic pathogen species, their pathogens could still have a disproportionately high force of infection at the zoonotic interface, and some - such as Lassa virus in western Africa (Fichet-Calvet et al. 2007; Gibb et al. 2017) or rabies virus in Brazil (Rocha et al. 2017) - may still represent the most notable threats to biosecurity in urban and peri-urban environments.

Regardless of whether their greater known pathogen diversity comes from greater sampling, exposure, or immune factors, urban animals should be subject to expansive surveillance and management for known and unknown pathogens. Ultimately, a better understanding of the relative pathogen hazard residing within animals in wild, domestic, and urban contexts will allow us to further prioritise the design of sampling regimes, mitigating risk to human populations and allowing us to draw closer to identifying the relative roles of sampling and wildlife-to-human contact in generating perceived zoonotic risk.

\section{Acknowledgements}

This work was supported by funding to the Viral Emergence Research Initiative (VERENA) consortium including NSF BII 2021909 and a grant from Institut de Valorisation des Données (IVADO).

\section{Data availability}

Data and code used in this analysis are available at

https://github.com/gfalbery/UrbanOutputters.

\section{References}

Albery, G.F. \& Becker, D.J. (2021). Fast-lived Hosts and Zoonotic Risk. Trends Parasitol., 113.

Albery, G.F., Eskew, E.A., Ross, N. \& Olival, K.J. (2020). Predicting the global mammalian viral sharing network using phylogeography. Nat. Commun., 1-9.

Allen, T., Murray, K.A., Zambrana-Torrelio, C., Morse, S.S., Rondinini, C., Di Marco, M., et al. (2017). Global hotspots and correlates of emerging zoonotic diseases. Nat. Commun., 8, 1124.

Becker, D.J., Albery, G.F., Kessler, M.K., Lunn, T.J., Falvo, C.A., Czirják, G.Á., et al. (2019). Macroimmunology: the drivers and consequences of spatial patterns in wildlife immune defense. J. Anim. Ecol., 89, 972-995. 
Becker, D.J., Albery, G.F., Sjodin, A.R., Poisot, T. \& Dallas, T.A. (2020a). Predicting wildlife hosts of betacoronaviruses for SARS-CoV-2 sampling prioritization. bioRxiv, https://do.

Becker, D.J., Hall, R.J., Forbes, K.M., Plowright, R.K. \& Altizer, S. (2018). Anthropogenic resource subsidies and host-parasite dynamics in wildlife. Philos. Trans. R. Soc. $B$ Biol. Sci., 373.

Becker, D.J., Seifert, S.N. \& Carlson, C.J. (2020b). Beyond Infection: Integrating Competence into Reservoir Host Prediction. Trends Ecol. Evol.

Becker, D.J., Streicker, D.G. \& Altizer, S. (2015). Linking anthropogenic resources to wildlifepathogen dynamics: a review and meta-analysis. Ecol. Lett., 18, n/a-n/a.

Carlson, C.J., Albery, G.F., Merow, C., Trisos, C.H., Zipfel, C.M., Eskew, E.A., et al. (2020a). Climate change will drive novel cross-species viral transmission. bioRxiv.

Carlson, C.J., Dallas, T.A., Alexander, L.W., Phelan, A.L. \& Phillips, A.J. (2020b). What would it take to describe the global diversity of parasites? Proc. R. Soc. B Biol. Sci., 287, 20201841.

Chamberlain, S.A. \& Szöcs, E. (2013). taxize: taxonomic search and retrieval in R. F1000Research, 2, 191.

Chen, G., Li, X., Liu, X., Chen, Y., Liang, X., Leng, J., et al. (2020). Global projections of future urban land expansion under shared socioeconomic pathways. Nat. Commun., $11,1-12$.

Cohen, J.M., Sauer, E.L., Santiago, O., Spencer, S. \& Rohr, J.R. (2020). Divergent impacts of warming weather on wildlife disease risk across climates. Science (80-. )., 370, eabb1702.

Cummings, C.R., Khan, N.Y., Murray, M.M., Ellison, T., Welch, C.N., Hernandez, S.M., et al. (2020). Foraging in Urban Environments Increases Bactericidal Capacity in Plasma and Decreases Corticosterone Concentrations in White Ibises, 8, 1-11.

Downs, C.J., Dochtermann, N.A., Ball, R., Klasing, K.C. \& Martin, L.B. (2020a). The effects of body mass on immune cell concentrations of mammals. Am. Nat., 195.

Downs, C.J., Schoenle, L.A., Oakey, S.J., Ball, R., Jiang, R.H.Y., Klasing, K.C., et al. (2020b). Extreme hyperallometry of mammalian antibacterial defenses. bioRxiv, 2020.09.04.242107.

Fichet-Calvet, E., Lecompte, E., Koivogui, L., Soropogui, B., Doré, A., Kourouma, F., et al. (2007). Fluctuation of abundance and Lassa virus prevalence in Mastomys natalensis in Guinea, West Africa. Vector-Borne Zoonotic Dis., 7, 119-128.

Fritz, S.A., Bininda-Emonds, O.R.P. \& Purvis, A. (2009). Geographical variation in predictors of mammalian extinction risk: big is bad, but only in the tropics. Ecol. Lett., 12, 538549.

Gao, J. \& O'Neill, B.C. (2020). Mapping global urban land for the 21st century with datadriven simulations and Shared Socioeconomic Pathways. Nat. Commun., 11, 1-12.

Gibb, R., Moses, L.M., Redding, D.W. \& Jones, K.E. (2017). Understanding the cryptic nature of Lassa fever in West Africa. Pathog. Glob. Health.

Gibb, R., Redding, D.W., Chin, K.Q., Donnelly, C.A., Blackburn, T.M., Newbold, T., et al. (2020). Zoonotic host diversity increases in human-dominated ecosystems. Nature.

Guy, C., Thiagavel, J., Mideo, N. \& Ratcliffe, J.M. (2019). Phylogeny matters: Revisiting "a comparison of bats and rodents as reservoirs of zoonotic viruses." $R$. Soc. Open Sci., 6, 181182.

Hadfield, J.D. (2010). MCMC methods for multi-response generalized linear mixed models: 
the MCMCglmm R package. J. Stat. Softw., 33, 1-22.

Han, B.A., O'Regan, S.M., Paul Schmidt, J. \& Drake, J.M. (2020). Integrating data mining and transmission theory in the ecology of infectious diseases. Ecol. Lett., ele.13520.

Hanson, D.A., Britten, H.B., Restani, M. \& Washburn, L.R. (2007). High prevalence of Yersinia pestis in black-tailed prairie dog colonies during an apparent enzootic phase of sylvatic plague. Conserv. Genet., 8, 789-795.

Hassell, J.M., Begon, M., Ward, M.J. \& Fèvre, E.M. (2017). Urbanization and Disease Emergence: Dynamics at the Wildlife-Livestock-Human Interface. Trends Ecol. Evol., 32, 55-67.

Hulme-Beaman, A., Dobney, K., Cucchi, T. \& Searle, J.B. (2016). An Ecological and Evolutionary Framework for Commensalism in Anthropogenic Environments. Trends Ecol. Evol.

Hwang, J., Kim, Y., Lee, S.W., Kim, N.Y., Chun, M.S., Lee, H., et al. (2018). Anthropogenic food provisioning and immune phenotype: Association among supplemental food, body condition, and immunological parameters in urban environments. Ecol. Evol., 8, 30373046.

IUCN. (2019). The IUCN Red List of Threatened Species. IUCN Red List Threat. Species. Version 2019-2. Available at: https://www.iucnredlist.org. Last accessed .

Jones, K.E., Habib, M., Bielby, J., Boakes, E.H., Gittleman, J.L., Carbone, C., et al. (2009). PanTHERIA: a species-level database of life history, ecology, and geography of extant and recently extinct mammals. Ecology, 90, 2648-2648.

Jones, K.E., Patel, N.G., Levy, M. a, Storeygard, A., Balk, D., Gittleman, J.L., et al. (2008). Global trends in emerging infectious diseases. Nature, 451, 990-993.

Keesing, F., Belden, L.K., Daszak, P., Dobson, A., Harvell, C.D., Holt, R.D., et al. (2010). Impacts of biodiversity on the emergence and transmission of infectious diseases. Nature, 468, 647-652.

Lindgren, F. \& Rue, H. (2015). Bayesian Spatial Modelling with R-INLA. J. Stat. Softw., 63, $1-25$.

Lindgren, F., Rue, H. \& Lindstrom, J. (2011). An explicit link between Gaussian fields and Gaussian Markov random fields: the stochastic partial differential equation approach. $J$. R. Stat. Soc. B, 73, 423-498.

Lloyd-Smith, J.O., Cross, P.C., Briggs, C.J., Daugherty, M., Getz, W.M., Latto, J., et al. (2005). Should we expect population thresholds for wildlife disease? Trends Ecol. Evol., 20, 511-519.

Loh, E.H., Zambrana-Torrelio, C., Olival, K.J., Bogich, T.L., Johnson, C.K., Mazet, J.A.K., et al. (2015). Targeting Transmission Pathways for Emerging Zoonotic Disease Surveillance and Control. Vector-Borne Zoonotic Dis., 15, 432-437.

Masi, E., Pino, F.A., Santos, M. das G.S., Genehr, L., Albuquerque, J.O.M., Bancher, A.M., et al. (2010). Socioeconomic and environmental risk factors for urban rodent infestation in Sao Paulo, Brazil. J. Pest Sci. (2004)., 83, 231-241.

Mason, P. (1994). Parasites of deer in New Zealand. New Zeal. J. Zool., 21, 39-47.

Mollentze, N. \& Streicker, D.G. (2020). Viral zoonotic risk is homogenous among taxonomic orders of mammalian and avian reservoir hosts, 1-8.

Morse, S.S., Mazet, J. a K., Woolhouse, M., Parrish, C.R., Carroll, D., Karesh, W.B., et al. (2012). Prediction and prevention of the next pandemic zoonosis. Lancet, 380, 19561965. 
Murray, M.H., Sánchez, C.A., Becker, D.J., Byers, K.A., Worsley-Tonks, K.E.L. \& Craft, M.E. (2019). City sicker? A meta-analysis of wildlife health and urbanization. Front. Ecol. Environ., 17, 575-583.

Olival, K.J., Hosseini, P.R., Zambrana-Torrelio, C., Ross, N., Bogich, T.L. \& Daszak, P. (2017). Host and viral traits predict zoonotic spillover from mammals. Nature, 546, 646650.

Ostfeld, R.S., Levi, T., Jolles, A.E., Martin, L.B., Hosseini, P.R. \& Keesing, F. (2014). Life History and demographic drivers of reservoir competence for three tick-borne zoonotic pathogens. PLoS One, 9.

Plourde, B.T., Burgess, T.L., Eskew, E.A., Roth, T.M., Stephenson, N. \& Foley, J.E. (2017). Are disease reservoirs special? Taxonomic and life history characteristics. PLoS One, 12, e0180716.

Rocha, S.M., de Oliveira, S. V., Heinemann, M.B. \& Gonçalves, V.S.P. (2017). Epidemiological Profile of Wild Rabies in Brazil (2002-2012). Transbound. Emerg. Dis., 64, 624-633.

Santini, L., González-Suárez, M., Russo, D., Gonzalez-Voyer, A., von Hardenberg, A. \& Ancillotto, L. (2019). One strategy does not fit all: determinants of urban adaptation in mammals. Ecol. Lett., 22, 365-376.

Seto, K.C., Güneralp, B. \& Hutyra, L.R. (2012). Global forecasts of urban expansion to 2030 and direct impacts on biodiversity and carbon pools. Proc. Natl. Acad. Sci. U. S. A., 109, 16083-16088.

Shaw, L.P., Wang, A.D., Dylus, D., Meier, M., Pogacnik, G., Dessimoz, C., et al. (2020). The phylogenetic range of bacterial and viral pathogens of vertebrates. Mol. Ecol., 1-19.

Shipley, B. (2009). Confirmatory path analysis in a generalized multilevel context. Ecology, 90, 363-368.

Stephens, P.R., Pappalardo, P., Huang, S., Byers, J.E., Farrell, M.J., Gehman, A., et al. (2017). Global Mammal Parasite Database version 2.0. Ecology, 98, 1476.

Strandin, T., Babayan, S.A. \& Forbes, K.M. (2018). Reviewing the effects of food provisioning on wildlife immunity. Philos. Trans. R. Soc. B Biol. Sci., 373.

Wardeh, M., Risley, C., Mcintyre, M.K., Setzkorn, C. \& Baylis, M. (2015). Database of hostpathogen and related species interactions, and their global distribution. Sci. Data, 2, 150049.

Wells, K., Gibson, D.I., Clark, N.J., Ribas, A., Morand, S. \& McCallum, H.I. (2018). Global spread of helminth parasites at the human-domestic animal-wildlife interface. Glob. Chang. Biol., 24, 3254-3265.

Wells, K., Morand, S., Wardeh, M. \& Baylis, M. (2020). Distinct spread of DNA and RNA viruses among mammals amid prominent role of domestic species. Glob. Ecol. Biogeogr., 29, 470-481.

Werner, C.S. \& Nunn, C.L. (2020). Effect of urban habitat use on parasitism in mammals: a meta-analysis. Proceedings. Biol. Sci., 287, 20200397.

Wilman, H., J., B., J., S., C., de L.R., M., R. \& W, J. (2014). EltonTraits 1 . 0 $\square$ : Species-level foraging attributes of the world's birds and mammals. Ecology, 95, 2027. 